\title{
REPARACIÓN E IMPERMEABILIZACIÓN DE LAS JUNTAS DE LA PRESA DE CAVALLERS, EN LOS PIRINEOS DE LÉRIDA-ESPAÑA
}

\author{
(REPAIR AND WATERPROOFING OF JOINTS AT THE CAVALLERS DAM IN \\ THE PYRENNEES OF LERIDA-SPAIN)
}

M. Carbonell De Masy, Licenciado en Ciencias Químicas. Director de la División Polímeros Líquidos de TEXSA, S. A.

\section{RESUMEN}

La patologia del hormigón armado y su reparación, no cabe duda que es un tema de actualidad a nivel mundial.

En este informe se expone la problemática planteada en el hormigón armado de una presa de contrafuertes, en los Pirineos de Lérida, que ocasionaba una pérdida de agua de $60 \mathrm{l} / \mathrm{seg}$ undo, la solución técnica dada y su proceso de ejecución a temperaturas del orden de $0^{\circ} \mathrm{C}$.

Una parte de la reparación fue llevada a cabo en la primavera de 1984 y, el resto, a principios de 1985. Los años

transcurridos están confirmando la eficacia del tratamiento.

\section{SUMMARY}

The problems with reinforced concrete and their repair are undoubtedly of great interest nowadays throughout the world.

The problems of the reinforced concrete of the buttress dam in the Catalonian Pyrenees are considered in this article.

Said problems led to a loss of 60 liters of water/sec. The technical solution to the problem is explained, as well as how it was carried out at temperatures of around $\mathrm{O}^{\circ} \mathrm{C}$.

Part of the repair work was done in the Spring of 1984, and the remainder at the beginning of 1985. The years which have elapsed confirm the effectiveness of the treatment.

\section{INTRODUCCIÓN}

La presa de Cavallers fue construida a finales de los 50 , con el fin de embalsar principalmente aguas procedentes del deshielo.

El sistema de construcción fue el de contrafuertes. Entre cada pareja de éllos se formó una junta vertical que aguas arriba se subdividía en dos, separadas por una estructura de hormigón armado de sección pentagonal, de acuerdo con la figura 1, en el que se ha marcado el deterioro superficial.

La impermeabilización de la junta, según planos, se había basado fundamentalmente en la pletina de acero galvanizado adosado a la junta, y en la masilla asfáltica aplicada posteriormente para rellenar todo el espacio hasta el encuentro con la viga pentagonal.

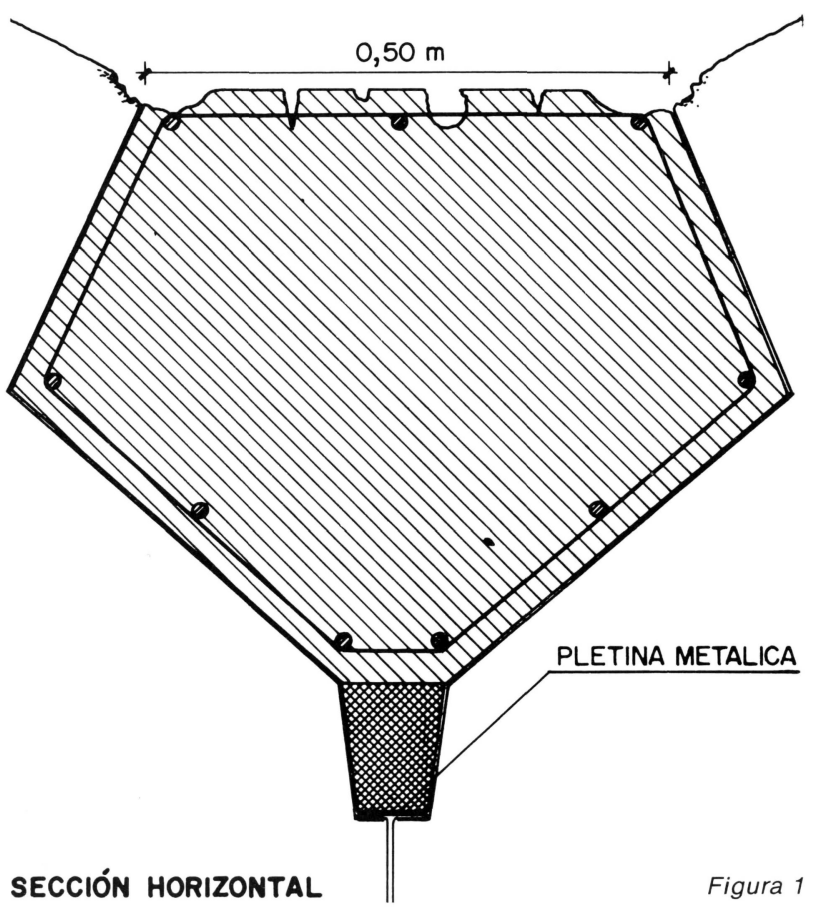

http://informesdelaconstruccion.revistas.csic.es 


\section{PATOLOGÍA DE LA PRESA}

Transcurridos treinta años de la explotación de la presa, ha sido necesario acometer su reparación ante las elevadas pérdidas de agua por filtraciones que ascendian a $60 \mathrm{l} /$ segundo y, naturalmente, relacionado con lo anterior, a la degradación, cada año más intensa, de la estructura pentagonal.

Entre las causas del deterioro de dicha estructura destaca, en primer lugar, la penetración de un agua de gran pureza procedente del deshielo por las fisuras existentes, con la correspondiente solubilización del hormigón. Ello unido a la carbonatación del hormigón que rodea las armaduras, ha ocasionado la oxidación de éstas y la consiguiente destrucción del hormigón que las recubre.

Otra segunda causa importante, hay que atribuirla a los procesos destructivos de los ciclos hielo-deshielo.

Las fisuras existentes en la obra eran de dos tipos:

- Por una parte, las longitudinales a la junta y que procedían de la separación existente entre la viga pentagonal y el resto del hormigón de la presa. En un principio estuvieron selladas superficialmente con una masilla asfáltica que una vez envejecida dejó de funcionar.

- Por otra parte, las transversales a la viga pentagonal correspondientes a las juntas de trabajo.

Una vez iniciada la destrucción del hormigón, el camino para la desaparición de la estanquidad estaba abierto.

\section{SOLUCIÓN TÉCNICA}

Para resolver la problemática anteriormente expuesta, la Dirección Técnica de ENHER se decidió por una solución basada en tres tipos de actuaciones que se encuentran ilustradas en la figura 2 :

a) Sustitución del hormigón deteriorado por un nuevo material de relleno de mayor resistencia y que no tuviera retracción de fraguado.

b) Sellado de las juntas verticales a ambos lados de la viga pentagonal que garantizasen su estanquidad.

c) Protección de la superficie de la viga pentagonal incluyendo las juntas y el hormigón correspondiente a las mismas en un ancho de $20 \mathrm{~cm}$.

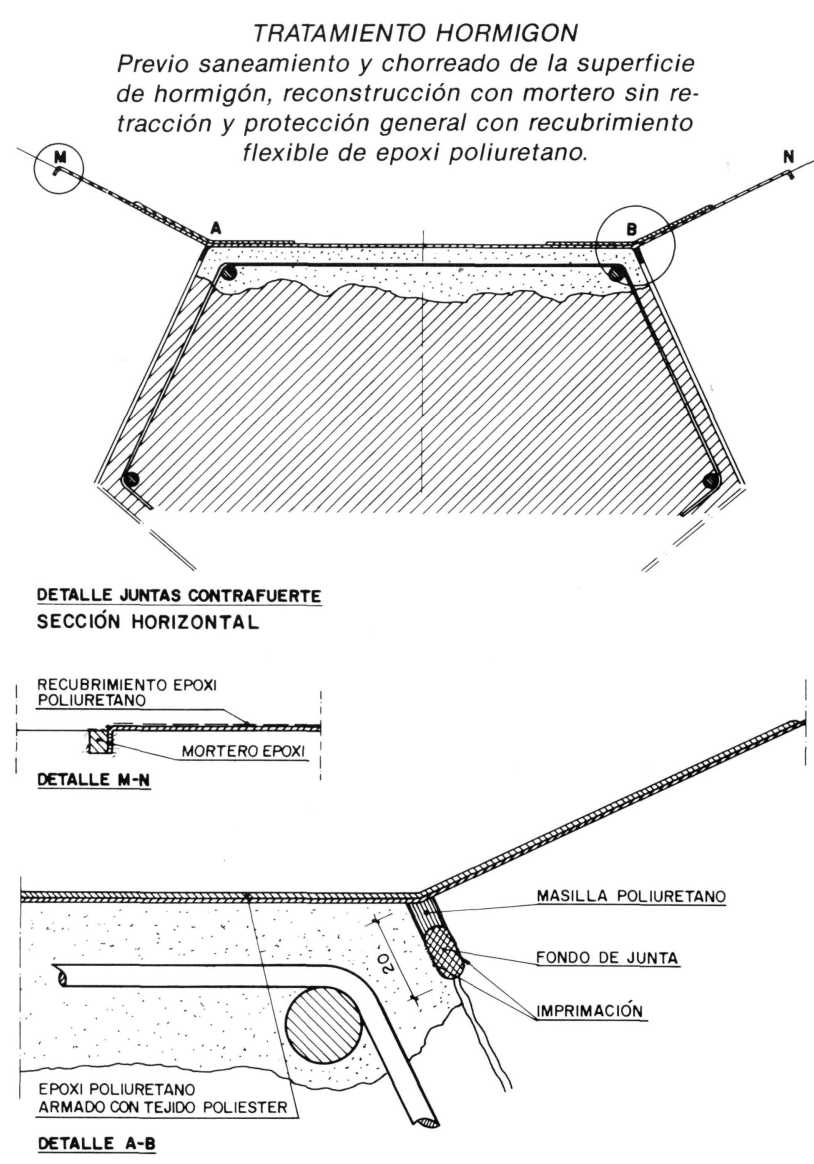

Figura 2

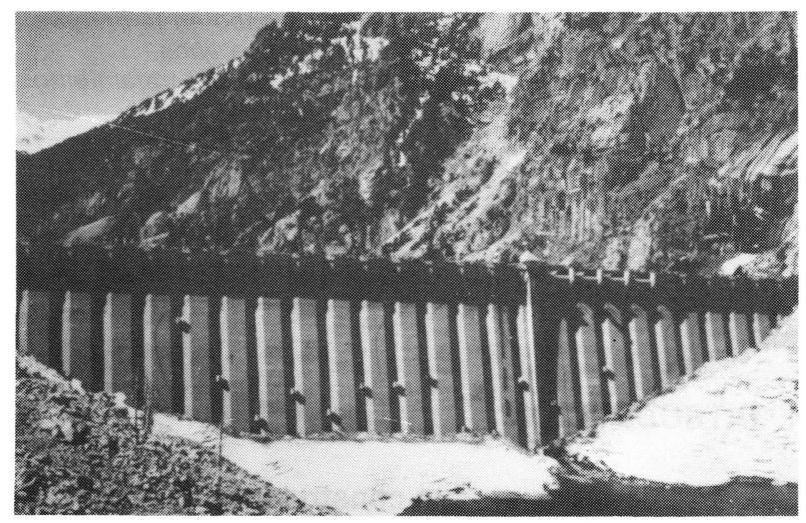

Foto n. ${ }^{\circ}$ 1.-Vista general de la presa aguas arriba.

Es de destacar que la obra tenía que realizarse entre el 15 de marzo y el 15 de mayo, con el fin de aprovechar al máximo el agua del deshielo para la producción de energía eléctrica. Ello obligaba a trabajar a temperaturas de hasta $8^{\circ} \mathrm{C}$ bajo cero y a estar expuestos, además, a lluvias y a nevadas (Foto n. ${ }^{\circ} 1$ ).

\subsection{Morteros de relleno}

Una vez saneado el soporte y tratado con chorro de arena, el espesor de material a reponer era muy variable, oscilando entre $20 \mathrm{~mm}$ e incluso más de $20 \mathrm{~cm}$. 
A tenor de las condiciones en que habia que trabajar $y$ de la necesidad de finalizar en poco tiempo las tres fases de la solución técnica dada, el mortero de relleno debería cumplir, entre otras, las siguientes condiciones:

a) Endurecimiento a temperaturas del orden de $0^{\circ} \mathrm{C}$ consiguiendo alta resistencia en pocos días.

b) No tener retracción de fraguado.

c) No necesitar para su curado la presencia de humedad ni la aplicación de líquidos de curado.

d) Resistencia al ciclo hielo-deshielo.

e) Poderse adherir aunque hubiera humedad.

f) Poseer tal compacidad y resistencia que pudiera conservar el máximo de tiempo su integridad.

Ante estas exigencias y, sobre todo, las indicadas en los apartados $c, b$ y $c$, quedaban descartados los materiales basados en cemento Portland o aluminosos. De los restantes morteros a base de ligante inorgáni$\mathrm{co}$, los que mejor podían satisfacer los requisitos expuestos eran los basados en cementos de fosfato de magnesio que no sólo no tienen retracción, sino que incluso se expanden y pueden endurecer hasta $20^{\circ} \mathrm{C}$ bajo cero. Otra ventaja es que pueden adherirse sobre soportes húmedos. La única limitación del mortero de fosfato de magnesio era su espesor, ya que debía ser superior a $20 \mathrm{~mm}$.

Para espesores inferiores a $30 \mathrm{~mm}$ una mejor adherencia se conseguiría con morteros a base de ligantes orgánicos, ya que previamente se aplicaba una imprimación del ligante sin carga. Se estudiaron cuatro tipos de morteros poliméricos a base de las siguientes resinas reactivas: epoxi, poliuretano, poliéster no saturado y metacrilato de metilo.

Por su mejor adherencia, menor retracción y otras cualidades se decidió un mortero a base de resinas epoxi endurecible a partir de $3^{\circ} \mathrm{C}$ y con un bajo módulo de elasicidad a compresión a bajas temperaturas.

La única precaución que debía tenerse en cuenta era que el soporte estuviera seco, lo cual era fácil por debajo de $0^{\circ} \mathrm{C}$. Para temperaturas superiores era necesario desviar el agua que afluía a las juntas.

Una gran ventaja en el empleo del mortero epoxi es que seguidamente se podía aplicar el revestimiento protector.

(c) Consejo Superior de Investigaciones Científicas Licencia Creative Commons 3.0 España (by-nc)

\subsection{Sellado de las juntas verticales}

La elección del sistema de sellado correspondió a una masilla de poliuretano de los componentes endurecibles a partir de $5^{\circ} \mathrm{C}$, previa aplicación de una imprimación a los bordes que asegurase una buena adherencia.

Antes de sellar se colocaba como fondo de junta un perfil de espuma de polietileno.

\subsection{Revestimiento protector}

Entre los requisitos que debía cumplir destacamos los siguientes:

a) Capacidad de absorber los movimientos de las juntas anteriormente selladas, actuando así como segunda estanquidad.

b) Máxima resistencia a la abrasión y a la intemperie.

c) Máxima impermeabilidad a la penetración de agua con que estaba en contacto, y la suficiente permeabilidad al vapor de agua a fin de mantener una buena adherencia sobre soportes que contuvieran algo de humedad.

De todos los requisitos, este último era el más difícil de cumplir. La humedad del soporte aludido no era só. lo la que pudiera tener retenido el mortero de fosfato de magnesio en los primeros días de su ejecución sino, en general, la humedad que pudiera llegar al interior del hormigón y que luego, durante el proceso de secado, tuviera que "respirar" a través del revestimiento protector.

Partiendo de la necesidad de una protección de máxima durabilidad era necesario un revestimiento con un espesor superior a $1 \mathrm{~mm}$ y de ahí, la necesidad de tener que basarse en resinas reactivas sin disolvente.

Los revestimientos basados sólo en poliepoxis tenían el inconveniente de una elevada impermeabilidad al vapor de agua y, con ello, el riesgo de desprendimiento.

Los revestimientos basados sólo en poliuretanos tenían como desventaja, frente a los poliepoxi, una menor adherencia.

A la vista de los datos teóricos y de los resultados prácticos en laboratorio, se decidió por una formulación mixta epoxi-poliuretano. El poliuretano le confería, además, la flexibilidad necesaria para absorber los movimientos de las juntas. 
Para facilitar, aún más, el "puenteado" sobre la junta se reforzaba el revestimiento con un tejido de poliéster no tejido. Para aumentar aún más la resistencia del revestimiento frente a la radiación ultravioleta y a la abrasión, se pensó en aplicar una pintura reactiva de poliuretano alifático, pero dadas las dificultades ambientales para su correcta aplicación, tuvo que sustituirse por una pintura epoxi emulsionada en agua.

ENHER quiso experimentar antes en obra esta solución. En la primavera de 1983, se hizo una primera prueba en una zona de la pantalla de la presa de Mequinenza, a través de cuyas juntas de trabajo penetraba agua.

Ante sus buenos resultados, incluso con hormigón húmedo, en el invierno de 1984 se realizaron unas pruebas en la presa de Cavallers, en dos juntas, cuyo resultado fue también positivo. A la vista de ello, se dio la conformidad para proceder en la primavera de 1985 a la reparación e impermeabilización de las 25 juntas restantes.

\section{EJECUCIÓN}

Para poder realizar el tratamiento fue necesario, en primer lugar, hacer unos pozos de unos $4 \mathrm{~m}$ en las bases de las juntas, con el fin de poder acceder a las zonas de contacto hormigón-roca. Lo primero que tuvo que eliminarse fue la capa de hielo de aproximadamente $50 \mathrm{~cm}$ de espesor.

La excavación se tuvo que realizar a mano, y fue necesario fracturar a base de martillo picador y cuñas las piedras de gran tamaño. En la mayoría de los pozos fue necesario entibar, debido a la profundidad y a la poca estabilidad del terreno cuando empezó a deshelar.

En la foto.$^{\circ} 2$, puede observarse un pozo. Antes de iniciarse la limpieza con chorro de arena, una bomba extrae el agua que queda en el pozo.

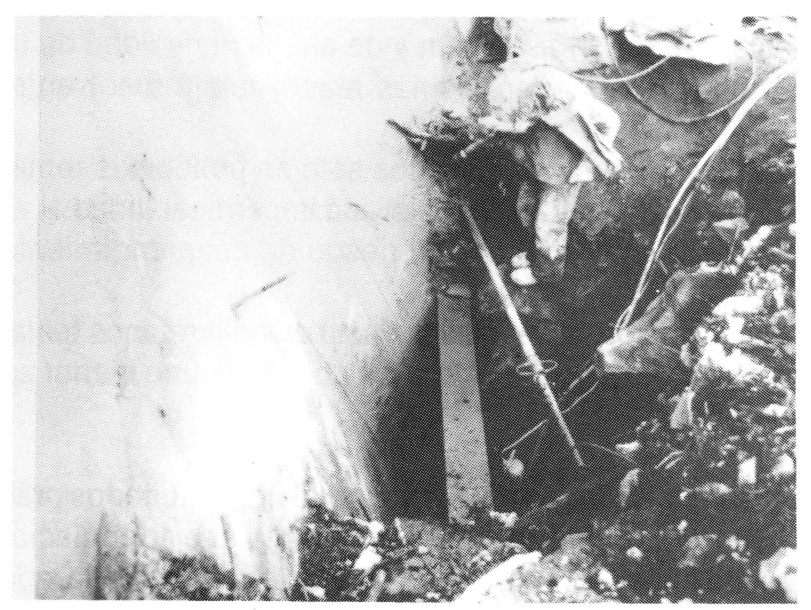

Foto n. ${ }^{\circ}$ 2.-Vista de uno de los pozos antes de iniciar el chorreado de la viga.

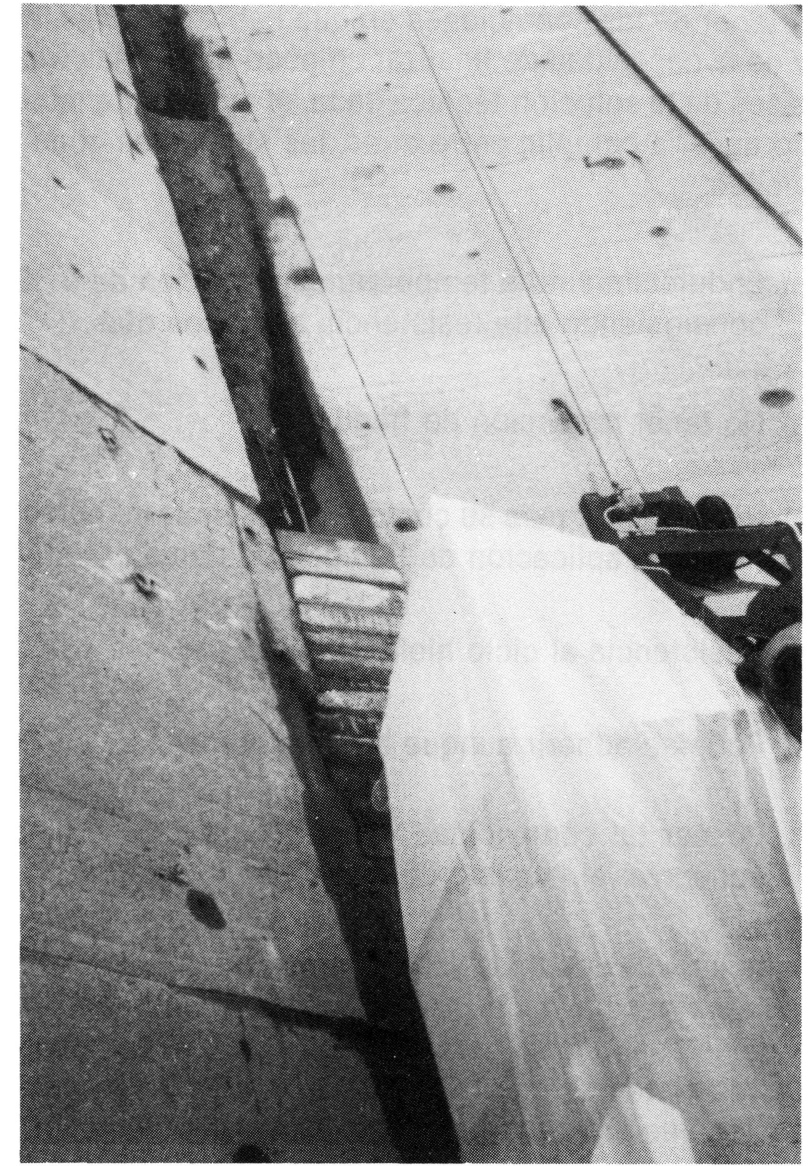

Foto n. ${ }^{\circ}$ 3.- En algunas zonas la viga pentagonal habia quedado prác ticamente destruida en su totalidad.

El resto del proceso de trabajo ha consistido en las siguientes fases:

4.1. Saneado del hormigón de la viga pentagonal y zonas adyacentes, hasta un ancho máximo de $90 \mathrm{~cm}$. Antes del chorreado de arena hubo un repicado previo con pistolete neumático.

4.2. Relleno del hueco formado a base de dos tipos de materiales. Uno de ellos a base de fosfato de magnesio, utilizado, en general, para espesores mayores de $50 \mathrm{~mm}$ cuando se trabajaba con temperaturas por debajo de los $0^{\circ} \mathrm{C}$ o si el soporte presentaba humedad. El otro, a base de epoxi, era utilizado para rellenos de menor espesor y que requisieran un soporte seco. En ambos casos se colocaban, previamente, unas tablas de encofrado con el fin de llevar a cabo un buen compactado. (Foto n. ${ }^{\circ}$ )

En cuanto a la armadura, hubo que reponerla en alguna zona, bien por estar rota o muy disminuido su diámetro.

http://informesdelaconstruccion.revistas.csic.es 


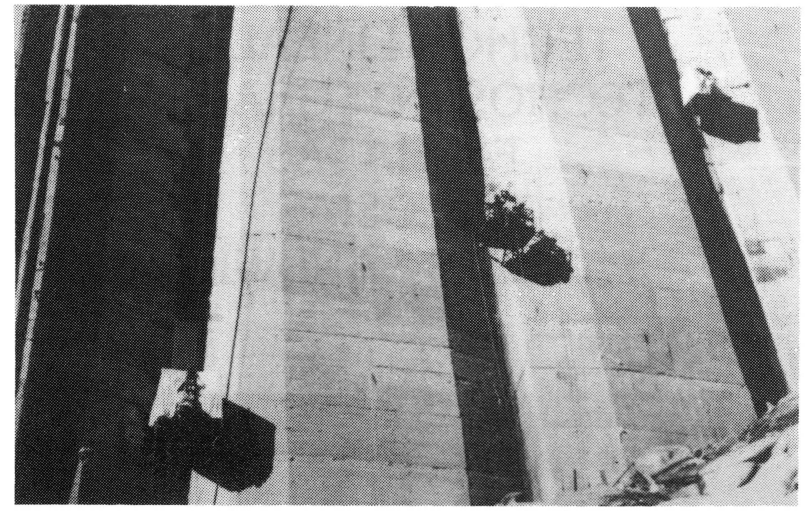

Foto n. ${ }^{\circ}$ 4.-Aplicación del revestimiento epoxi-poliuretano armado Detalle del proceso de reparación.

El total de litros de mortero de reposición as. cendió a 28.200, lo que representa una media de $22,67 \mathrm{l} / \mathrm{m}$ lineal.

4.3. Perfilado de las juntas verticales con máquinas de disco y sellado interior, con una masilla de poliuretano, previa colocación del fondo de junta y, anteriormente, haber dado imprimación (Detalle A-B).

4.4. Refuerzo del sellado indicado anteriormente mediante la aplicación de una capa de epoxipoliuretano, armado con un "no tejido", en un ancho de $20 \mathrm{~cm}$.

Una vez endurecido el citado refuerzo, se vuelve a dar otra capa de epoxi-poliuretano sobre el total de $90 \mathrm{~cm}$ sobre el cual se impregna una banda del "no tejido", entregándose la lámina elástica en unas zonas laterales que se sellan con masilla epoxi (Detalle M-N). (Foto n. ${ }^{\circ}$ ).

Antes de iniciar la aplicación del revestimiento se chorreó el mortero de relleno ejecutado con el fin de favorecer la adherencia de éste.

4.5. Finalmente se aplicó una capa de pintura epoxi (dos componentes), emulsionada en agua.

\section{RESULTADOS}

La solución expuesta, además de regenerar y proteger el hormigón armado deteriorado, ha logrado reducir el total de pérdidas de agua de $60 \mathrm{l} / \mathrm{segundo}$, a menos de $10 \mathrm{l} / \mathrm{segundo}$, con embalse lleno. Para la eliminación total haría falta tratar todas las grietas de la pantalla, así como las uniones con cimentación y drenajes.

Han transcurrido aproximadamente 4 años desde la reparación, y por ahora no se observa ninguna anomalía.

\section{BIBLIOGRAFÍA}

- INTERNATIONAL COMMISSION ON LARGE DAMS. "Synthetic resins for facing of dams". Bulletin 43, 1982.

- TEXSA (División Polímeros Líquidos). "Aplicaciones de los compuestos epoxi en la construcción”. Ed. Cotexsa. Barcelona, 1984

- E. HERRERO, A. ROTGER, L. DOMENE. "Regeneración de las juntas de dilatación de la presa de contrafuertes de Cavallers". Primeras jornadas españolas de presas. Madrid, 1985.

- E. DRENIER. "Utilisation des resines synthetiques dans l'entretien des barrages d'électricité de France". International Symposium adhesion between polymers and concrete. Aix-en Provence 1986.

- F. J. BUNDIES. "Adhesion of modern barrier coats on concrete motorway bridges". International Symposium adhesion between polymers and concrete. Aix-en Provence, 1986.

- TEXSA (División Polímeros Líquidos). "Patología del hormigón debido a la oxidación de la armadura. Reparación y prevención con polímeros líquidos". Ed. Cotexsa. Barcelona, 1986

- K. H. HENTSCHEL. "Flexible epoxy resin system" $54^{\circ}$ Congreso de GDCH, Group Paints. Bad Kissingen. Alemania, 1987 